\title{
Segment Dynamics in Thin Polystyrene Films Probed by Single-Molecule Optics
}

\author{
Nikodem Tomczak, ${ }^{\dagger, \ddagger}$ Renaud A. L. Vallée, ${ }^{\dagger, \neq}$ Erik M. H. P. van Dijk, ${ }^{\ddagger}$ Laurens Kuipers, ${ }^{\ddagger}$ \\ Niek F. van Hulst, $\neq$ and G. Julius Vancso*, \\ Department of Materials Science and Technology of Polymers and Applied Optics Group, University of Twente, \\ MESA+ Institute for Nanotechnology, Faculty of Science and Technology, P.O. Box 217, 7500 AE Enschede,
} The Netherlands

Received October 27, 2003; E-mail: g.j.vancso@ct.utwente.nl

Polymers have recently been demonstrated to exhibit different chain dynamic behavior, as compared to the bulk, when confined into the geometry of ultrathin films. ${ }^{1,2}$ Investigations of the glass transition temperature $\left(T_{\mathrm{g}}\right)$ of thin, supported, polystyrene films by ellipsometry, ${ }^{3}$ Brillouin light scattering, ${ }^{4}$ dielectric spectroscopy, ${ }^{5}$ or positron annihilation lifetime spectroscopy (PALS), ${ }^{6}$ among others, showed large $T_{\mathrm{g}}$ depressions. This behavior is thought to be due to the existence of a surface layer where the polymer chain dynamics is enhanced over that in the bulk. This layer becomes dominant for very thin films, thereby shifting $T_{\mathrm{g}}$ of the films toward lower temperatures. The existence of such a surface layer is also supported by measurements of $T_{\mathrm{g}}$ for free-standing polymer films ${ }^{7}$ and by a direct examination of the polymer surface by PALS. ${ }^{8}$ However, all experimental techniques mentioned provide ensembleaveraged information. Furthermore, the depth at which deviation between the surface and the bulk dynamics becomes significant could not be addressed in detail due to the lack of depth resolution of these techniques on the nanometer scale.

Single molecule fluorescence detection (SMD) intrinsically avoids ensemble averaging. In combination with high spatial and temporal resolution, SMD is an ideal tool for investigating structure and dynamics of the probe environment on the nanoscale. Through the use of different approaches, SMD has already been employed with success to study macromolecular systems ${ }^{9,10}$ and inorganic/ organic composite films. ${ }^{11}$ In a recent study ${ }^{12}$ we have introduced a new single-molecule approach based on fluorescent lifetime fluctuations to give direct insight into local, nanoscale dynamics of the polymer matrix surrounding a chromophoric probe on the segmental level.

In this communication we report, for the first time, on the use of single-molecule fluorescence lifetime to investigate thin film effects in a glassy polymer system at temperatures far below bulk $T_{\mathrm{g}}$. We find that the dynamics of the surroundings of the probe becomes enhanced when the constituent macromolecules are confined into a thin film. The characteristic film thickness at which this behavior becomes noticeable is several times larger than the radius of gyration $\left(R_{\mathrm{g}}\right)$ of the polymer chain used. Our result points toward the existence of interfacial regions with enhanced dynamics and represents the first step toward depth-resolved studies of polymer films on the nanoscale using SMD.

Thin-film samples were prepared by spin coating DiD $\left(1,1^{\prime}\right.$ dioctadecyl-3,3,3',3'-tetramethylindodicarbocyanine, Molecular Probes D-307)/polystyrene (PS, $M_{\mathrm{n}}=89300 \mathrm{~g} / \mathrm{mol}, M_{\mathrm{w}} / M_{\mathrm{n}}=1.06$, Polymer Standard Service) solutions onto cleaned glass cover slides. The films were subsequently annealed, first for $12 \mathrm{~h}$ at $60^{\circ} \mathrm{C}$ and later for $3 \mathrm{~h}$ at $105^{\circ} \mathrm{C}$ in order to remove the residual solvent and relax all stresses induced by the spin coating procedure. The

$\dagger$ Department of Materials Science and Technology of Polymers.

¥ Applied Optics Group. a)
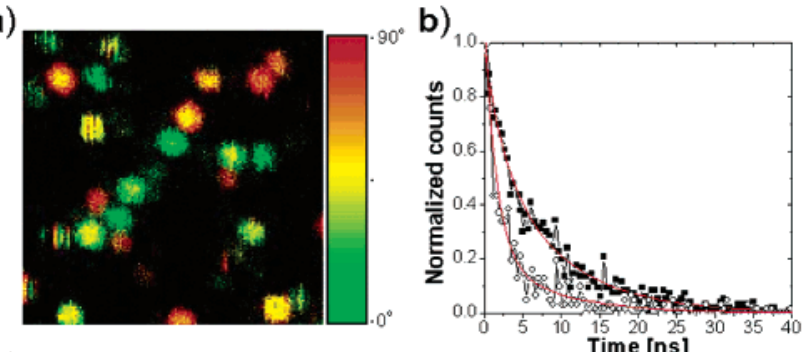

c)

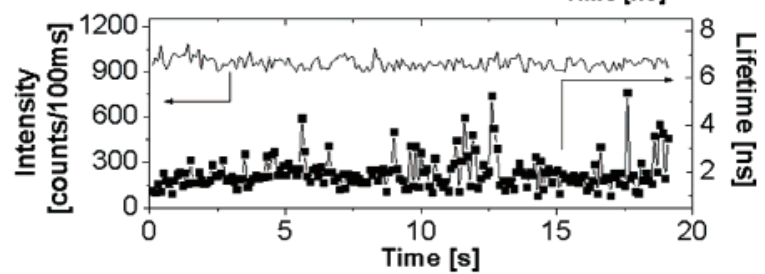

Figure 1. (a) $5 \times 5 \mu \mathrm{m}^{2}$ fluorescence intensity scan of single DiD molecules in a $110 \mathrm{~nm}$ thick PS film at $22^{\circ} \mathrm{C}$. The color scale indicates the polarization of the fluorescence, which is a measure for the in-plane orientation of the molecules. Constant color confirms that the molecules are not rotating in the matrix. (b) Fluorescence decays of one DiD molecule from which a short $(2.5 \mathrm{~ns}, 0)$ and long $(6 \mathrm{~ns}$, fluorescence lifetime was extracted. The red lines correspond to single-exponential fits to the data. (c) Fluorescence intensity and lifetime traces for a single DiD in PS. Excursions to longer lifetimes (up to $5 \mathrm{~ns}$ ) are clearly visible.

concentration of the dye was $10^{-9} \mathrm{M}$ in the resulting PS films, and the thickness was varied between 10 and $200 \mathrm{~nm}$. A NanoScope III Atomic Force Microscope (Digital Instruments, Santa Barbara) was used to estimate the planarity and the roughness of the samples $(<2 \mathrm{~nm})$ before and after the measurements, respectively. No dewetting of the thin films was observed at any stage. To detect the individual molecules within the polymeric matrix, we used a scanning fluorescence confocal microscope (custom built) equipped with suitable dichroic, excitation, and emission filters. A picosecond pulsed laser (PicoQuant, 800-B) was employed to excite the molecules at the wavelength of $635 \mathrm{~nm}$. Fluorescence photons were collected by two avalanche photodetectors (SPCM-AQ-14, EG\&G Electro Optics) placed after a polarization beam splitter. An SPC 500 time-correlated single-photon counting card (Becker \& Hickl) was used for time-resolved lifetime measurements. ${ }^{12}$ For higher temperature measurements, a custom-built hot stage was placed below the sample and the temperature was monitored with an accuracy of $\pm 2{ }^{\circ} \mathrm{C}$. For each film thickness, 400 probe molecules were analyzed for two different temperatures, 22 and $35{ }^{\circ} \mathrm{C}$, respectively.

Figure 1a depicts a $5 \times 5 \mu \mathrm{m}^{2}$ fluorescence intensity scan. Characteristic on-off behavior and single-step photobleaching observed confirm that the spots on the image correspond to single emitters. For each single molecule, we collected intensity and 


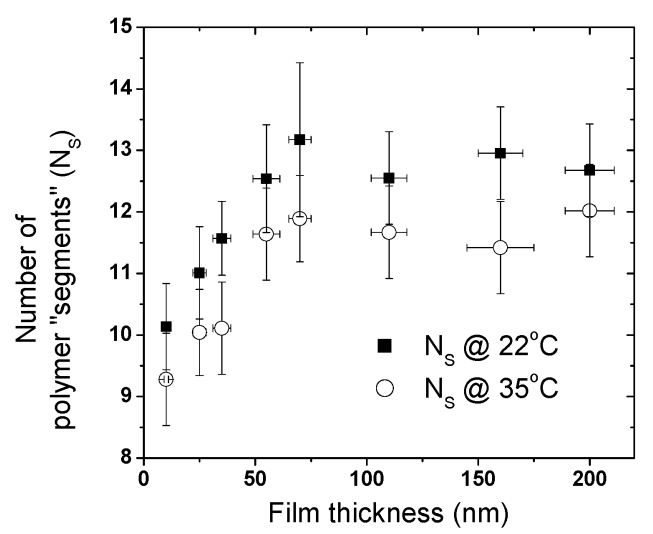

Figure 2. Mean value of the effective number of polymer "segments" in the rearrangement volume around the single-molecule probes as a function of PS film thickness for two different temperatures.

fluorescence lifetime traces with $100 \mathrm{~ms}$ time intervals, during 20$60 \mathrm{~s}$ total observation time. The accuracy of lifetime determination is on the order of 300 ps. Examples of decay curves (two snapshots at different times for the same molecule) are presented in Figure 1b. In Figure 1c, fluorescence intensity and lifetime traces are shown. Changes in the lifetime as a function of time for the same molecule are clearly visible. From the lifetime trajectory of each molecule we built lifetime distributions, which were fitted with a distribution function. ${ }^{13}$ From the fitting parameters we determine the effective number of polymer "segments" $\left(N_{S}\right)$ taking part in the rearrangement volume around the single probe using the method described in detail in ref $12 . N_{\mathrm{S}}$ determined by this procedure provides direct information concerning local, segmental scale polymer dynamics and its spatial distribution. The value of $N_{\mathrm{S}}$ is very sensitive for small density fluctuations around the probe within the probed time interval. Higher values of $N_{\mathrm{S}}$ can be visualized as a larger collection of polymer segments, which must move cooperatively during segmental relaxation around the probe, therefore indicating slower system dynamics.

In Figure 2, we present the mean of $N_{\mathrm{S}}$ values for single molecules as a function of PS film thickness for two different temperatures. The effect of temperature on $N_{\mathrm{S}}$ is consistent with our earlier results showing a decrease of $N_{\mathrm{S}}$ with increasing temperature. ${ }^{12}$ The behavior of $N_{\mathrm{S}}$ as a function of film thickness can be divided into two regions. One region can be assigned to film thickness above a certain threshold value $h_{0}$, where $N_{\mathrm{S}}$ remains essentially constant. Below $h_{0}, N_{\mathrm{S}}$ becomes a function of thickness and decreases with decreasing film thickness. The transition $\left(h_{0}\right)$ between the two regions is estimated from the graph to be around $50-60 \mathrm{~nm}$.

Two main effects are known to be responsible for the observed decrease of $N_{\mathrm{S}}$ with decreasing film thickness: chain confinement (when the characteristic length scale of the polymer chain is comparable to the dimensions of the system) and the presence of interfaces with modified dynamics. However, for the molar mass of the polymer used here, the average radius of gyration $\left(R_{\mathrm{g}}\right)$ of the macromolecules is $9-10 \mathrm{~nm}$ assuming a chain conformation as in the $\theta$ state, i.e., 6 times smaller than the value of $h_{0}$. Thus, chain confinement is not expected to play a major role in our case. For a similar system consisting of air/polystyrene/glass, it was reported that the polymer/glass interface does not perturb the film dynamics. ${ }^{14}$ Therefore, we attribute the decrease of $N_{\mathrm{S}}$ for small film thickness to modified polymer dynamics induced by the presence of a free polymer surface. ${ }^{15}$ We also conclude that in such thin films, the dynamics of the polymer is modified far below the bulk glass transition temperature $\left(T_{\mathrm{g}, \mathrm{b}}=100{ }^{\circ} \mathrm{C}\right.$ as measured by DSC) even though a large fraction of the macromolecules cannot "sense" the possible effect of the interfaces nor are they forced to adopt a different conformation due to the confinement represented by the thin film. Apparently due to the connectivity and interpenetration of polymer chains, surface-induced enhancement of polymer dynamics propagate into the sample. The temperature dependence of $N_{\mathrm{S}}$ for PS films yields $\mathrm{d} N_{\mathrm{S}} / \mathrm{d} T=-0.11$ [segments/deg] ${ }^{12}$ thus the dynamics in the thinnest film is equivalent to the dynamics in thicker films (thickness $>h_{0}$ ) if it was observed at temperatures elevated by as much as $30{ }^{\circ} \mathrm{C}$.

From the mean lifetime of a collection of single molecules as a function of film thickness (not presented here), we rule out any dye segregation to one of the interfaces or at a certain depth within the samples. Also, from the stability of the fluorescence polarization state and from the mean fluorescence lifetime for each molecule during the observation time, we excluded the possibility that the decrease of $N_{\mathrm{S}}$ is caused by an increased rotational or translational activity of the probe in thinner films at the experimental temperatures (65 and $80{ }^{\circ} \mathrm{C}$ below $T_{\mathrm{g}, \mathrm{b}}$, respectively) that could cause lifetime fluctuations ${ }^{16}$ and thus influence $N_{\mathrm{S}}$.

In conclusion, we used a single-molecule lifetime technique to study the influence of the film thickness on local polymer dynamics on the nanometer length scale at temperatures far below the glass transition temperature. We find modified segment scale dynamics when the polymer is confined into films with thicknesses below $50-60 \mathrm{~nm}$ corresponding to 6 times the radius of gyration. In the future, we intend to extend our investigations to a controlled, depthresolved dynamics study in thin polymer films from the surface to the bulk using NSOM-based approaches.

Acknowledgment. The Council for Chemical Sciences of the Netherlands Organization for Scientific Research (NWO-CW) is gratefully acknowledged for financial support. E.v.D. is financed by FOM, Dutch Foundation for Fundamental Research of Matter.

Supporting Information Available: Representative histograms of the width parameter and the corresponding $N_{\mathrm{S}}$ distributions (PDF). This material is available free of charge via the Internet at http://pubs.acs.org.

\section{References}

(1) Jones, R. A. L. Curr. Opin. Colloid. Interface. Sci. 1999, 4, 153-158.

(2) Forrest, J. A.; Dalnoki-Veress, K. Adv. Colloid Interface Sci. 2001, 94 $167-196$.

(3) Keddie, J. L.; Jones, R. A. L.; Cory, R. A. Europhys. Lett. 1994, 27 $59-64$.

(4) Forrest, J. A.; Dalnoki-Veress, K.; Dutcher, J. R. Phys. Rev. E 1997, 56, $5705-5716$.

(5) Fukao, K.; Miyamoto, Y. Phys. Rev. E 2001, 64, Art. No. 011803.

(6) DeMaggio, G. B.; Frieze, W. E.; Gidley, D. W.; Zhu, M.; Hristov, H. A ; Yee, A. F. Phys. Rev. Lett. 1997, 78, 1524-1527.

(7) Forrest, J. A.; Dalnoki-Veress, K.; Stevens, J. R.; Dutcher, J. R. Phys. Rev. Lett. 1996, 77, 2002-2005.

(8) Cao, H.; Zhang, R.; Yuang, J. P.; Huang, C. M.; Jean, Y. C.; Suzuki, R.; Ohdaira, T.; Nielsen, B. J. Phys.: Condens. Matter 1998, 10, 1042910442 .

(9) Dickson, R. M.; Norris, D. J.; Tzeng, Y. L.; Moerner, W. E. Science 1996, $274,966-969$.

(10) Deschenes, L. A.; Vanden Bout, D. A. Science 2001, 292, 255-258.

(11) Bardo, A. M.; Collinson, M. M.; Higgins, D. A. Chem. Mater. 2001, 13 , $2713-2721$

(12) Vallée, R. A. L.; Tomczak, N.; Kuipers, L.; Vancso, G. J.; van Hulst, N. F. Phys. Rev. Lett. 2003, 91, Art. No. 038301.

(13) Experimental data are best fitted with a gamma distribution function in the form of: $\gamma(\tau)=\beta \tau^{(\alpha-1)} \mathrm{e}^{-\beta \tau}$.

(14) Keddie, J. L.; Jones, R. A. L.; Cory, R. A. Faraday Discuss. 1995, 98, $219-230$.

(15) Mansfield, K. F.; Theodorou, D. N. Macromolecules 1991, 24, 62836294.

(16) Vallée, R. A. L.; Tomczak, N.; Gersen, H.; van Dijk, E. M. H. P.; García Parajó, M. F.; Vancso, G. J.; van Hulst, N. F. Chem. Phys. Lett. 2001, $348,161-167$.

JA039249H 